\title{
INFLUENCIA DEL VERMICOMPOST EN EL CRECIMIENTO DE LAS PLANTAS. APORTES PARA LA ELABORACIÓN DE UN CONCEPTO OBJETIVO
}

\author{
Jorge Domínguez, Cristina LazCano \& María Gómez-Brandón \\ Departamento de Ecología y Biología Animal. Universidad de Vigo. Vigo E-36310, España. E-mail: \\ jdguez@uvigo.es, cristina@uvigo.es, mariagomez@uvigo.es
}

Domínguez, J., C. Lazcano \& M. Gómez-Brandón. 2010. Influencia del vermicompost en el crecimiento de las plantas. Aportes para la elaboración de un concepto objetivo. Acta Zoológica Mexicana (n.s.), Número Especial 2: 359-371.

RESUMEN. Numerosos estudios han demostrado que la adición de humus de lombriz a los sustratos de cultivo tiene efectos beneficiosos sobre el crecimiento y desarrollo de gran cantidad de especies vegetales. Los mecanismos a través de los que se produce esta estimulación incluyen el aporte de nutrientes, la mejora de las propiedades físicas del sustrato y el aporte de microorganismos beneficiosos para el desarrollo vegetal capaces de aumentar la disponibilidad de nutrientes y producir sustancias con efectos fitohormonales. En los últimos años se ha dedicado un importante esfuerzo a la investigación de estos efectos hormonales, y más concretamente al papel de las sustancias húmicas como portadoras de sustancias reguladoras del crecimiento vegetal (PGRs). Sin embargo, la existencia de efectos discordantes en la literatura pone de manifiesto la necesidad de abrir nuevas vías de investigación así como de definir de forma más objetiva y clara el concepto de vermicompost.

Palabras clave: Sustancias reguladoras del crecimiento vegetal, rizosfera, microorganismos, sustancias húmicas, agroecología, fertilizantes orgánicos.

Domínguez, J., C. Lazcano \& M. Gómez-Brandón. 2010. Influence of vermicompost on plant growth. Contributions toward the development of an objective concept. Acta Zoológica Mexicana (n.s.), Número Especial 2: 359-371.

ABSTRACT. Many studies have shown that the addition of vermicompost to plant growing media has beneficial effects on growth and development of a wide range of plant species. Some of the proposed mechanisms involved in this growth stimulation are: nutrient release, enhancement of physical properties of the substrates and inoculation of beneficial microorganisms with the ability to increase the solubility of essential nutrients for plant growth and produce plant growth promoting substances. In the last years research efforts have been centered in the investigation of these hormonal effects and, more precisely, on the role of humic substances as carriers of these plant growth regulating substances (PGRs). However, the existence of conflicting results in the literature evidences the need for new research lines and definition of a clear and objective concept of vermicompost.

Key words: Plant growth regulating substances, rhizosphere, microorganisms, humic substances, agroecology, organic fertilizers.

Recibido: 16/05/2008; aceptado: 08/01/2010. 


\section{INTRODUCCIÓN}

\section{Efectos de las lombrices en la fertilidad de los suelos}

Los efectos beneficiosos de las lombrices de tierra en la fertilidad del suelo se conocen desde hace tiempo. A lo largo de los últimos cincuenta años, han sido numerosos los ejemplos que han demostrado que tanto en poblaciones naturales, como en poblaciones introducidas artificialmente, las lombrices pueden tener efectos significativos sobre el crecimiento vegetal. Los ejemplos más relevantes han sido ilustrados en los trabajos de Lee (1985), Edwards et al. (1995), Edwards \& Bohlen (1996), Edwards (1998) y Brown et al. (1999). Otros trabajos como los de van Rhee (1965), Atlavinyte (1974), Edwards \& Lofty $(1976,1980)$, Atlavinyte \& Vanagas (1982) y Stephens \& Davoren (1995), demuestran que la adición de lombrices al suelo produce un incremento en el crecimiento de varias especies de cereales en zonas templadas. Más recientemente mediante una serie de experimentos de campo, se ha demostrado que la inoculación de lombrices en terrenos agrícolas tropicales puede tener también efectos notables sobre el crecimiento y rendimiento vegetal (Spain et al. 1992, Brown et al. 1999, Lavelle \& Spain 2001).

Entre los efectos beneficiosos de las lombrices se incluyen la mejora de algunas propiedades físicas del suelo como la estructura (Jongmans et al. 2003), la turbación (Edwards 1998), la capacidad de retención de agua, el drenaje y la formación y degradación de agregados (Edwards \& Bohlen 1996), así como efectos químicos y biológicos en la degradación de la materia orgánica y en el ciclado de nutrientes (Edwards \& Bohlen 1996, Edwards 1998). Todos estos procesos contribuyen de forma fundamental a la fertilidad del suelo y por lo tanto al crecimiento vegetal y a la productividad de los cultivos.

A pesar de que los microorganismos constituyen una fuente muy importante de alimento para las lombrices (Edwards \& Fletcher 1988), la biomasa de bacterias, hongos y actinomicetes suele ser mayor en las deyecciones frescas de las lombrices que en el suelo circundante (Edwards \& Bohlen 1996). Estos aumentos podrían deberse al incremento en las poblaciones microbianas tras el paso por el intestino de las lombrices, bien debido a que el alimento que seleccionan constituye un sustrato más rico para el desarrollo microbiano, o bien por que la fragmentación de la materia orgánica en sus molleja incrementa la superficie disponible para el ataque microbiano (Dkhar \& Mishra 1986, Tiwari \& Mishra 1993). Se ha demostrado además que las lombrices pueden incrementar la actividad y la biomasa microbiana del suelo (Schindler-Wessels et al. 1996). Existen numerosas evidencias científicas que demuestran que las lombrices estimulan considerablemente la descomposición de la materia orgánica, tanto durante el paso a través de sus intestinos como en el proceso de envejecimiento de las deyecciones (Scheu 1987, Daniel \& Anderson 1992, Aira et al. 2003, 2007).

La aceleración de la descomposición de la materia orgánica inducida por las lombrices de tierra, especialmente las epigeas, ha sido aprovechada para el tratamiento 
de residuos orgánicos como residuos animales, agrícolas, urbanos e industriales y su transformación en fertilizantes orgánicos. Las lombrices se encargan de fraccionar el sustrato orgánico estimulando la actividad microbiana e incrementando las tasas de mineralización, de forma que el residuo orgánico se transforma rápidamente en un substrato humificado cuya textura y tamaño de partícula son mucho más finas que las de los compost termofílicos tradicionales. Estos procesos han sido detallados por Edwards (1998) y Domínguez (2004).

De la misma forma que hay estudios que ponen de manifiesto los efectos positivos de las lombrices sobre el suelo y las plantas, también hay bastantes estudios que demuestran que el empleo de vermicompost puede producir también efectos positivos, evidenciando así los efectos indirectos de las lombrices sobre las plantas.

En los últimos años, y debido a la creciente demanda de productos de agricultura ecológica en el mercado, se ha producido un incremento considerable en la producción y la aplicación de este tipo de abonos orgánicos. Al mismo tiempo también ha aumentado la investigación acerca de los posibles efectos beneficiosos sobre el crecimiento vegetal y los mecanismos responsables. Este artículo presenta una revisión de las evidencias científicas de los efectos del vermicompost en el crecimiento de las plantas con el fin de aportar una visión global y objetiva y contribuir al desarrollo de nuevas vías de investigación.

\section{Efectos del vermicompost en el crecimiento de las plantas}

Se ha demostrado que la adición del vermicompost a los suelos y sustratos de cultivo incrementa considerablemente el crecimiento y la productividad de una gran cantidad de cultivos hortícolas tales como el tomate (Atiyeh et al. 1999, Atiyeh et al. 2000a, Atiyeh et al. 2000b, Atiyeh et al. 2001, Hashemimajd et al. 2004, Gutiérrez-Miceli et al. 2007, Steffen et al., ese número), la lechuga (Steffen et al., ese número), los pimientos (Arancon et al. 2004a), los ajos (Argüello et al. 2006), las fresas (Arancon et al. 2004b), algunas plantas medicinales (Prabha et al. 2007), algunas leguminosas como el garbanzo verde (Karmegam et al. 1999), algunas gramíneas como el sorgo (Reddy \& Ohkura 2004, Sunil et al. 2005) y el arroz (Bhattacharjee et al. 2001), algunas hierbas aromáticas como la albahaca (Anwar et al. 2005), algunos frutales como el plátano (Cabanas Echevarria et al. 2005) y la papaya (Acevedo \& Pire 2004), y algunas plantas ornamentales como los geranios (Chand et al. 2007), los tajetes (Atiyeh et al. 2002a), las petunias (Arancon et al. 2008), los crisantemos (Hidalgo \& Harkess 2002a) y las flores de pascua (Hidalgo \& Harkess 2002b).

A diferencia de los fertilizantes minerales, el vermicompost constituye una fuente de nutrientes de liberación lenta, que se van poniendo a disposición de la planta a medida que ésta los va necesitando (Chaoui et al. 2003). Además, la adición de ver- 
micompost puede producir una mejora significativa en las propiedades físicas tanto de los sustratos artificiales de cultivo (Hidalgo \& Harkess 2002a, b) como del suelo (Marinari et al., 2000, Atiyeh et al., 2001, Bhattacharjee et al., 2001, Ferreras et al., 2006).

Por otra parte la aparición de otros trabajos ha puesto de manifiesto que los efectos del vermicompost podrían no reducirse a los meramente físicos y/o químicos y señalan la posible existencia de mecanismos biológicos de estimulación del crecimiento vegetal. Scott (1988) observó que la adición de pequeñas dosis de vermicompost al medio de cultivo de las especies ornamentales Chaemocyparis lawsonian, Elaeagnus pungens, Cuppressocypari leylandii, Phyracantha spp., Cotoneaster conspicus y Viburnum bodnantense producía incrementos significativos en el crecimiento respecto a un medio control suplementado con una dosis de nutrientes equivalente. Edwards \& Burrows (1988) señalaron que el crecimiento de varias especies ornamentales, cultivadas en los sustratos originados tras el procesamiento de residuos orgánicos por la especie de lombriz Eisenia fetida, fue mucho mayor de lo esperable para ser causado únicamente por la modificación en la disponibilidad de nutrientes por la acción de las lombrices. Señalaron además que estos efectos se mantenían aún cuando el vermicompost era diluido en proporción 20:1 con otros materiales de cultivo, y el contenido de nutrientes era igualado al de los fertilizantes minerales. Además, el patrón de crecimiento de las plantas, que incluía alteraciones en el desarrollo foliar, en la elongación de la raíz y del tallo, y floración, apuntaba a la posible existencia de algún factor biológico distinto al del aporte de nutrientes, como la producción de sustancias capaces de influenciar el crecimiento vegetal (ácidos húmicos, enzimas libres), como responsables de estos efectos.

\section{Producción de sustancias reguladoras del crecimiento vegetal}

Parece claro que la rápida descomposición de los residuos orgánicos que llevan a cabo las lombrices con los microorganismos, da lugar a sustratos con una actividad y diversidad microbianas considerablemente mayores que las del residuo de partida, llegándose a multiplicar varias veces los valores iniciales (Aira et al. 2007). Este incremento de la actividad microbiana además de aumentar la tasa de transformación de nutrientes a formas más fácilmente asimilables por las plantas, puede afectar al crecimiento vegetal mediante el incremento en la actividad enzimática, la supresión de enfermedades y la producción de sustancias reguladoras del crecimiento, ó PGRs (Plant Growth Regulating Substances) como se ha venido demostrando últimamente.

A lo largo de los últimos años se han aportado gran cantidad de pruebas que demuestran que los microorganismos, incluyendo algas, levaduras, actinomicetos, hongos y bacterias son capaces de producir PGRs tales como auxinas, giberelinas, citoquininas, etileno y ácido abscísico en cantidades apreciables (Arshad \& Frankem- 
berger 1993, Frankemberger \& Arshad 1995). Muchos de los microorganismos que se pueden encontrar en la rizosfera son capaces de producir tales sustancias (Vessey 2003). Barea et al. (1976) encontraron que de 50 cepas bacterianas aisladas de la rizosfera de distintas especies vegetales, un $86 \%$ era capaz de producir auxinas, un $58 \%$ giberelinas, y un $90 \%$ sustancias con actividad kinetina. Existen gran cantidad de trabajos que demuestran la producción de sustancias reguladoras del crecimiento vegetal por poblaciones mixtas de microorganismos del suelo, pero sin embargo existe poca información acerca de su disponibilidad para las plantas, su persistencia y degradación en el suelo, así como de sus efectos en el crecimiento vegetal (Arshad \& Frankemberger 1993, Frankemberger \& Arshad 1995).

Algunos trabajos demuestran que las sustancias reguladoras del crecimiento vegetal pueden ser tomadas por las plantas en cantidades suficientes como para producir cambios en su crecimiento. Algunos ejemplos incluyen el aumento del crecimiento de gramíneas por auxinas producidas por Azospirillum brasilense (Kucey 1983, Barbieri et al. 1986) o la promoción del crecimiento de la caña de azúcar por auxinas producidas por rizobacterias del género Enterobacter (Mirza et al. 2001). Existe una evidencia creciente de que las giberelinas de origen microbiano pueden alterar el crecimiento y desarrollo vegetal. Recientemente, Gutiérrez-Mañero et al. (2001) demostraron que determinados extractos de las rizobacterias Bacillus pumilus y Bacillus licheniformis contenían giberelinas capaces de estimular el crecimiento de mutantes enanos de alisos. Por otra parte, el incremento en el vigor de las plántulas de algunas especies vegetales ha sido atribuido a la producción de citoquininas por los microorganismos Arthrobacter y Bacillus spp. en el suelo (Inbal \& Feldman 1982, Jagnow 1987).

Se ha sugerido que las lombrices podrían ser agentes importantes capaces de influenciar la producción de sustancias promotoras del crecimiento vegetal por los microorganismos mediante la estimulación y promoción de la actividad microbiana tanto en suelos como en sustratos orgánicos (Nielson 1965, Springett \& Syers 1979, Graf \& Makeschin 1980, Tomati et al. 1983, Grappelli et al. 1987, Dell'Agnola \& Nardi 1987, Nardi et al. 1988, Tomati et al. 1987, 1988, 1990, Tomati \& Galli 1995). Krishnamoorty \& Vajranabhiah (1986) demostraron, mediante experimentos llevados a cabo con varias poblaciones de lombrices, que siete de las especies estudiadas eran capaces de incrementar la producción de auxinas y citoquininas en residuos orgánicos. Además mostraron que existía una fuerte correlación entre las poblaciones de lombrices y la cantidad de auxinas y citoquininas encontradas en 10 suelos de cultivo diferentes. Estos autores señalaron que ambas sustancias podían persistir en el suelo hasta 10 semanas, aunque podían ser degradadas en pocos días si eran expuestas a la luz solar.

Existen otros autores que sostienen que las lombrices son las responsables de la producción de sustancias con efecto hormonal. El primero en sugerir esta teoría fue 
Gavrilov (1963), y esta teoría fue reforzada por la primera prueba de la presencia de PGRs en tejidos de Aporrectodea caliginosa, Lumbricus rubellus, y E. fetida, aportada por Nielson (1965), el cual aisló sustancias indólicas de las lombrices y observó un incremento significativo en el crecimiento de plantas de guisante debido a la adición de extractos de las lombrices. Nielson extrajo además una sustancia de las especies Aporrectodea longa, Lumbricus terrestris, y Dendrobaena rubidus, capaz de estimular el crecimiento vegetal; sin embargo, sus experimentos no excluían la posibilidad de que dichas sustancias procediesen de los microorganismos existentes en el intestino y tejidos de las lombrices.

Graff \& Makeschin (1980) estudiaron los efectos de sustancias producidas por L. terrestris, A. caliginosa y E. fetida en la producción de biomasa de la ballica (Lolium sp.). Añadieron los lixiviados del riego de macetas que contenían lombrices a macetas sin lombrices, y concluyeron que ciertas sustancias capaces de influenciar el crecimiento vegetal, ó PGIs (Plant Growth Influencing Substances), habían sido liberadas en el suelo por las tres lombrices, aunque no siguieron especulando acerca de la naturaleza de estas sustancias. Más recientemente, El Harti et al. (2001a, 2001b) mostraron que un extracto bruto de la lombriz L. terrestris era capaz de estimular la rizogénesis en semillas de haba debido a la presencia de compuestos indólicos de origen endógeno. Tomati et al. (1983, 1987, 1988), Tomati et al. (1990), Grapelli et al. (1987) y Tomati \& Galli (1995) observaron que los incrementos en el crecimiento de varias especies ornamentales y champiñones al aplicar vermicompost de diferentes especies eran mucho mayores de lo que cabía esperar por el mero aporte de nutrientes contenidos en las dosis de humus aportadas. Además los efectos sobre el crecimiento incluían la estimulación del enraizado, cambios en el tiempo de floración y el alargamiento de los entrenudos. Mediante la comparación del crecimiento de Begonia, Petunia y Coleus, tras la adición de extractos acuosos de vermicompost o auxinas, giberelinas y citoquininas, concluyeron que existía gran evidencia de efectos hormonales originados por la actividad de las lombrices.

\section{El papel de las sustancias húmicas como estimuladoras del crecimiento vegetal}

El vermicompost originado a partir de residuos ganaderos, restos de comida, lodos de depuradora ó lodos de la industria papelera, contiene gran cantidad de sustancias húmicas (Atiyeh et al. 2002b, Canellas et al. 2002, Arancon et al. 2002). Los estudios realizados acerca de los efectos de estas sustancias sobre el crecimiento vegetal, bajo condiciones de nutrición mineral adecuada, han mostrado resultados muy positivos (Chen \& Aviad 1990, Hayes \& Wilson 1997). Las sustancias húmicas incrementaron de forma significativa la producción de biomasa de plántulas de maíz y avena (Lee \& Bartlett 1976, Albuzio et al. 1994), el número y la longitud de raíces en plantas 
de tabaco (Mylonas \& Mccants 1980), la biomasa de tallos, raíces y nódulos en soja, cacahuete, y trébol (Tan \& Tantiwiramanond 1983), el crecimiento vegetativo en plantas de chicoria (Valdrighi et al. 1996) y la inducción de la formación de tallos y raíces de ciertos cultivos tropicales en cultivo in vitro (Goenadi \& Sudharama 1995). El tratamiento de células de zanahoria con sustancias húmicas extraídas de las deyecciones de $A$. rosea aumentó el crecimiento celular e indujo cambios morfológicos similares a los inducidos por las auxinas (Muscolo et al. 1999).

La adición de sustancias húmicas a los sustratos de cultivo produce una curva de crecimiento típica de una respuesta hormonal. A concentraciones bajas, el aporte de sustancias húmicas produce un incremento proporcional en el crecimiento de la planta, mientras que a concentraciones mayores se observa una disminución del crecimiento. Este mismo patrón se ha observado tras la adición de vermicompost a sustratos de cultivo fertilizados con cantidades abundantes de los nutrientes esenciales (Atiyeh et al. 1999, 2000a, b, c, d).

En los últimos años se ha investigado la actividad biológica de las sustancias húmicas, especialmente de las derivadas de las deyecciones de las lombrices de tierra (Dell'Agnola \& Nardi 1987, Nardi et al. 1988, Muscolo et al. 1993). Las sustancias húmicas están formadas por asociaciones supramoleculares de moléculas orgánicas relativamente pequeñas agrupadas principalmente por enlaces hidrofóbicos. Estos complejos moleculares pueden disociarse dando lugar a fracciones de mayor ó menor peso molecular. Se ha comprobado que la actividad biológica se encuentra ligada a las fracciones de menor peso molecular (Dell'Agnolla \& Nardi 1987) que son las que más fácilmente se absorben y alcanzan la pared celular de las plantas (Nardi et al. 2002). Estas fracciones, en las que se han encontrado adsorbidas ciertas fitohormonas como el ácido indol acético (Quaggiotti et al. 2004, Canellas et al. 2002), son capaces de inducir la formación y el crecimiento de raíces laterales por medio de la activación de las bombas de $\mathrm{H}^{+}$-ATPasa de la membrana celular y de las vacuolas (Canellas et al. 2002, Zandonadi et al. 2006), así como de incrementar la absorción de nutrientes como los nitratos, mediante la activación de la trascripción de genes responsables de su transporte y metabolismo (Quaggiotti et al. 2004).

\section{Apuntes críticos y necesidades futuras en la investigación del efecto del vermicompost en el crecimiento vegetal}

A la luz de estas evidencias científicas parece bastante claro que el vermicompost es un complejo constituido por una amalgama de deyecciones de lombriz (incluyendo metabolitos propios de las especies utilizadas), materia orgánica humificada y microorganismos, cuya adición a los medios de cultivo es capaz de incrementar la germinación, el crecimiento, la floración, la fructificación y la resistencia a patógenos de una gran cantidad de especies vegetales. Esta estimulación en el desarrollo y creci- 
miento podría estar causada por la acción de varios mecanismos tales como el aporte gradual de nutrientes, la mejora de las propiedades físicas del sustrato y el aporte de microorganismos beneficiosos para el desarrollo vegetal capaces de aumentar la disponibilidad de nutrientes y de producir una diversa gama de sustancias con acción hormonal.

Algunos trabajos recientes muestran que los efectos del vermicompost pueden variar dependiendo de la especie vegetal considerada e incluso de la variedad (Zaller 2007), así como del material de partida, proceso de producción del vermicompost, tiempo de almacenamiento, y tipo de sustrato al que se vaya a incorporar (Rodda et al. 2006, Roberts et al. 2007). Además Lores et al. (2006) demostraron que en función de la especie de lombriz empleada y del residuo de partida, las comunidades microbianas originadas tras el vermicompostaje eran diferentes, lo que podría implicar distintas capacidades para estimular el crecimiento de las plantas.

A pesar de que la mayor parte de las investigaciones desarrolladas hasta el momento se han centrado en el estudio de los efectos hormonales, estas evidencias científicas ponen de manifiesto la complejidad de las interacciones vermicompost-planta así como la ausencia de un concepto claro y objetivo de vermicompost. El desarrollo de investigaciones en este sentido supondría una contribución importante tanto para la agricultura ecológica, acusada de graves carencias científicas (Trewavas 2001), como para la ecología del suelo, permitiendo desenmarañar algo más la compleja trama de las relaciones entre las plantas y el suelo.

\section{AGRADECIMIENTOS}

Este trabajo fue apoyado por la Xunta de Galicia (07MRU023383PR). Cristina Lazcano ha sido financiada por una beca de la Fundación "La Caixa". María GómezBrandón ha sido financiada por una beca FPU del Ministerio Español de Educación y Ciencia.

\section{LITERATURA CITADA}

Acevedo, I. C. \& R. Pire. 2004. Effects of vermicompost as substrate amendment on the growth of papaya (Carica papaya L.). Interciencia. 29(5): 274-279.

Aira, M., F. Monroy \& J. Domínguez. 2003. Effects of two species of earthworms (Allolobophora spp.) on soil systems: a microfaunal and biochemical analysis. Pedobiologia. 47: 877-881.

Aira, M., F. Monroy \& J. Domínguez. 2007. Earthworms strongly modify microbial biomass and activity triggering enzymatic activities during vermicomposting independently of the application rates of pig slurry. Science of the Total Environment. 385: 252-261

Albuzio, A., G. Concheri, S. Nardi \& G. Dell'Agnola. 1994. Effect of humic fractions of different molecular size on the development of oat seedlings grown in varied nutritional conditions. Pp. 199204. In: N. Senesi and T. M. Mianom (Eds.). Humic substances in the global environment and implications on human health. Elsevier Science, Amsterdam. 
Anwar, M., D. D. Patra, S. Chand, A. Kumar, A. A. Naqvi \& S. P. S. Khanuja. 2005. Effect of organic manures and inorganic fertilizer on growth, herb and oil yield, nutrient accumulation, and oil quality of French basil. Communications in Soil Science and Plant Analysis. 36(1-14): 1737-1746.

Arancon, N. Q., C. A. Edwards, A. Babenko, J. Cannon, P. Galvis \& J. D. Metzger. 2008. Influences of vermicomposts, produced by earthworms and microorganisms from cattle manure, food waste and paper waste, on the germination, growth and flowering of petunias in the greenhouse. Applied Soil Ecology. 39: 91-99.

Arancon, N. Q., C. A. Edwards, P. Bierman, C. Welch, \& J. D. Metzger. 2004b. Influences of vermicomposts on field strawberries: 1. effects on growth and yields. Bioresource Technology. 93: 145-153.

Arancon, N. Q., C. E. Edwards, R. M. Atiyeh \& J. D. Metzger. 2004a. Effects of vermicompost produced from food waste on the growth and yields of greenhouse peppers. Bioresource Technology. 93: 139-144.

Arancon, N. Q., S. Lee, C. A. Edwards \& R. M. Atiyeh. 2002. Effects of humic acids and aqueous extracts derived from cattle, food and paper-waste vermicomposts on growth of greenhouse plants. Pedobiologia. 47: 741-744.

Argüello, J. A., A. Ledesma, S. B. Núñez, C. H. Rodríguez \& M. D. C. Díaz Goldfarb. 2006. Vermicompost effects on bulbing dynamics nonstructural carbohydrate content, yield, and quality of 'Rosado Paraguayo' garlic bulbs. Hortscience. 41(3): 589-592.

Arshad, M., \& W. T. Frankenberger Jr. 1993. Microbial production of plant growth regulators. Pp. 307-347. In: F. B. Metting Jr. (Ed.). Soil microbial ecology: applications in agricultural and environmental management. Marcell Dekker, New York.

Atiyeh, R. M., N. Arancon, C. A. Edwards \& J. D. Metzger. 2000b. Influence of earthworm-processed pig manure on the growth and yield of greenhouse tomatoes. Bioresource Technology. 75: $175-180$.

Atiyeh, R. M., N. Arancon, C. A. Edwards \& J. D. Metzger. 2002a. The influence of earthwormprocessed pig manure on the growth and productivity of marigolds. Bioresource Technology. 81: 103-108.

Atiyeh, R. M., J. Dominguez, S. Subler \& C. A. Edwards. 2000c. Biochemical changes in cow manure processed by earthworms (Eisenia andrei) and their effects on plant-growth. Pedobiologia. 44: 709-724

Atiyeh, R. M., C. A. Edwards, S. Subler \& J. Metzger. 2000d. Earthworm processed organic wastes as components of horticultural potting media for growing marigolds and vegetable seedlings. Compost Science and Utilization. 8(3): 215-223.

Atiyeh, R. M., C. A. Edwards, S. Subler \& J. D. Metzger. 2001. Pig manure vermicompost as a component of a horticultural bedding plant medium: Effects on physicochemical properties and plant growth. Bioresource Technology. 78: 11-20.

Atiyeh, R. M., S. Lee, C. A. Edwards, N. Q. Arancon \& J. D. Metzger. 2002b. The influence of humic acids derived from organic wastes on plant growth. Bioresource Technology. 84: 7-14

Atiyeh, R. M., S. Subler, C. A. Edwards, G. Bachman, J. D. Metzger \& W. Shuster. 2000a. Effects of vermicomposts and compost on plant growth in horticultural container media and soil. Pedobiologia. 44: 579-590.

Atiyeh, R. M., S. Subler, C. A. Edwards \& J. Metzger. 1999. Growth of tomato plants in horticultural media amended with vermicompost. Pedobiologia. 43: 724-728.

Atlavinyte, O. 1974. Effect of earthworms on the biological productivity of barley. Institute of Zoology and Parasitology of the Academy of Sciences of Lithuania. I(65): 69-79. 
Atlavinyte, O. \& J. Vanagas. 1982. The effect of earthworms on the quality of barley and rye grain. Pedobiologia. 23: 256-262.

Barbieri, P., T. Zanelli, E. Galli \& G. Zanetti. 1986. Wheat inoculation with Azospirillum brasilense Sp6 and some mutants altered in nitrogen fixation and indole-3-aceticacid production. Microbiology Letters. 36: 87-90.

Barea, J. M., M. Navarro \& E. Montana. 1976. Production of plant growth regulators by rhizosphere phosphate-solubilizing bacteria. Journal of Applied Bacteriology. 40: 129-134.

Bhattacharjee, G., P. S. Chaudhuri \& M. Datta. 2001. Response of paddy (Var. TRC-87-251) crop on amendment of the field with different levels of vermicompost. Asian Journal of Microbiology, Biotechnology and Environmental Sciences. 3(3): 191-196.

Cabanas-Echevarría, M., A. Torres -García, B. Díaz-Rodríguez, E. F. H. Ardisana \& Y. CremeRamos. 2005. Influence of three bioproducts of organic origin on the production of two banana clones (Musa spp AAB.) obtained by tissue cultures. Alimentaria. 369: 111-116.

Canellas, L. P., F. L. Olivares, A. L. Okorokova \& A. R. Facanha. 2002. Humic acids isolated from earthworm compost enhance root elongation, lateral root emergence, and plasma H+-ATPase activity in maize roots. Plant Physiology. 130: 1951-1957.

Chand, S., P. Pande, A. Prasad, M. Anwar \& D. D. Patra. 2007. Influence of integrated supply of vermicompost and zinc-enriched compost with two graded levels of iron and zinc on the productivity of geranium. Communications in Soil Science and Plant Analysis. 38: 2581-2599.

Chaoui, H. I., L. M. Zibilske \& T. Ohno. 2003. Effects of earthworm casts and compost on soil microbial activity and plant nutrient availability. Soil Biology and Biochemistry. 35: 295-302.

Chen, Y. \& T. Aviad. 1990. Effects of humic substances on plant growth. Pp. 191-197. In: P. MacCarthy, C. E. Clapp, R. L. Malcolm and P. R. Bloom (Eds.). Humic substances in soil and crop sciences: selected reading. American Society of Agronomy, Madison.

Daniel, O. \& J. M. Anderson. 1992. Microbial biomass and activity in contrasting soil material after passage through the gut of the earthworm Lumbricus rubellus Hoffmeister. Soil Biology and Biochemistry. 24: 465-470.

Dell'Agnola, G. \& S. Nardi. 1987. Hormone-like effect and enhanced nitrate uptake induced by depolycondensed humic fractions obtained from Allolobophora rosea and A. caliginosa feces. Biology \& Fertility of Soils. 4: 115-118.

Dkhar, M. C. \& R. R. Mishra. 1986. Microflora in earthworm casts. Journal of Soil Biology and Ecology. 6: 24-31.

Domínguez, J. 2004. State of the art and new perspectives on vermicomposting research. Pp. 401-424. In: C. A. Edwards (Ed.). Earthworm ecology, $2^{\text {nd }}$ Ed. CRC Press, Boca Raton.

Edwards, C. A. 1998. Earthworm ecology. CRC/Lewis Press, Boca Raton.

Edwards, C. A., P. J. Bohlen, D. R. Linden \& S. Subler. 1995. Earthworms in agroecosystems. In: P. F. Hendrix (Ed.). Pp. 185-213. Earthworm ecology and biogeography in North America. CRC Press, Boca Raton.

Edwards, C. A. \& P. J. Bohlen. 1996. Biology and ecology of earthworms. Chapman and Hall, London.

Edwards, C. A. \& I. Burrows. 1988. The potential of earthworm composts as plant growth media. Pp. 211-219. In: C. A. Edwards and E. F. Neuhauser (Eds.). Earthworms in waste and environmental management. SPB Academic Publishing, The Hague.

Edwards, C. A. \& K. E. Fletcher. 1988. Interactions between earthworms and microorganisms in organic-matter breakdown. Agriculture, Ecosystems and Environment. 24: 235-247.

Edwards, C. A. \& J. R. Lofty. 1976. The influence of invertebrates on root crops grown with minimal or zero cultivation. Pp. 348-356. In: U. Lohm and T. Persson (Eds.). Soil organisms as components of ecosystems. Ecological Bulletins, vol. 25, Stockholm. 
Edwards, C. A. \& J. R. Lofty. 1980. Effects of earthworm inoculation upon the root growth of direct drilled cereals. Journal of Applied Ecology. 17: 533-543.

El Harti, A., M. Saghi, J-A. E. Molina \& G. Téller. 2001a. Production d'une substance rhizogéne à effet similaire à celui de l'acide indole acétique par le ver de terre Lumbricus terrestris. Canadian Journal of Zoology. 79: 1911-1920.

El Harti, A., M. Saghi, J-A. E. Molina \& G. Téller. 2001b. Production des composés indoliques rhizogénes par le ver de terre Lumbricus terrestris. Canadian Journal of Zoology. 79: 1921-1932.

Ferreras, L., E. Gomez, S. Toresani, I. Firpo \& R. Rotondo. 2006. Effect of organic amendments on some physical, chemical and biological properties in a horticultural soil. Bioresource Technology. 97: 635-640.

Frankenberger, W. T. Jr. \& M. Arshad. 1995. Phytohormones in soils: microbial production and function. Marcel Dekker, New York.

Gavrilov, K. 1963. Earthworms, producers of biologically active substances. Zhurnal Obshchei Biologii. 24: 149-54.

Goenadi, D. H. \& I. M. Sudharama. 1995. Shoot initiation by humic acids of selected tropical crops grown in tissue culture. Plant Cell Reports. 15: 59-62.

Graff, O.\& F. Makeschin. 1980. Beeinflussung des ertrags von weidelgras (Lolium multiflorum) durch ausscheidungen von regenwurmen dreier verschiedener arten. Pedobiologia. 20: 176-180.

Grappelli, A., E. Galli \& U. Tomati. 1987. Earthworm casting effect on Agaricus bisporus fructification. Agrochimica. 31 (4-5): 457-461.

Gutiérrez-Mañero, F. J., B. Ramos-Solano, A. Probanza, J. Mehouachi, F. R. Tadeo \& M. Talon. 2001. The plant-growth-promoting rhizobacteria Bacillus pumilus and Bacillus licheniformis produce high amounts of physiologically active gibberellins. Physiologia Plantarum. 111: 206-211.

Gutiérrez-Miceli, F. A., J. Santiago-Borraz, J. A. Montes Molina, C. C. Nafate, M. Abdud-Archila, M. A. Oliva Llaven, R. Rincón-Rosales \& L. Deendoven. 2007. Vermicompost as a soil supplement to improve growth, yield and fruit quality of tomato (Lycopersicum esculentum). Bioresource Technolology. 98: 2781-2786.

Hashemimajd, K., M. Kalbasi, A. Golchin \& H. Shariatmadari. 2004. Comparison of vermicompost and composts as potting media for growth of tomatoes. Journal of Plant Nutrition. 6: 1107-1123.

Hayes, M. H. B. \& W. S. Wilson. 1997. Humic substances, peats and sludges. RSC, Cambridge.

Hidalgo, P. R. \& R. L. Harkess. 2002a. Earthworm casting as a substrate amendment for Chrysanthemum production. Hortscience. 37(7): 1035-1039.

Hidalgo, P. R. \& R. L. Harkess. 2002b. Earthworm casting as a substrate for Poinsettia production. Hortscience. 37(2): 304-308.

Inbal, E., \& M. Feldman. 1982. The response of a hormonal mutant of common wheat to bacteria of the genus Azospirillium. Israel Journal of Botany. 31: 257-263.

Jagnow, G. 1987. Inoculation of cereal crops and forage grasses with nitrogen-fixing rhizosphere bacteria. Possible causes of success and failure with regard to yield response: A review. Zeitschrift für Pflanzenernährung und Bodenkunde. 150: 361-368.

Jongmans, A. G., M. M. Pulleman, M. Balabane, F. van Oort \& J. C. Y. Marinissen. 2003. Soil structure and characteristics of organic matter in two orchards differing in earthworm activity. Applied Soil Ecology. 24: 219-232.

Karmegam, N., K. Alagumalai \& T. Daniel. 1999. Effect of vermicompost on the growth and yield of green gram (Phaseolus aureus Roxb.). Tropical Agriculture. 76: 143-146.

Krishnamoorthy, R. V. \& S. N. Vajranabhiah. 1986. Biological activity of earthworm casts: An assessment of plant growth promoter levels in casts. Proceedings of the Indian Academy of Sciences (Animal Science). 95: 341-35. 
Kucey, R. M. N. 1983. Phosphate-solubilizing bacteria and fungi in various cultivated and virgin Alberta soils. Canadian Journal of Soil Science. 63: 671-678.

Lavelle, P. \& A. V. Spain. 2001. Soil ecology. Kluwer Academic, Dordrecht.

Lee, K.E. 1985. Earthworms: their ecology and relationships with soils and land use. Academic Press, Sydney.

Lee, Y. S. \& R. J. Bartlett. 1976. Stimulation of plant growth by humic substances. Soil Science Society of America Journal. 40: 876-879.

Lores, M., M. Gómez-Brandón, D. Pérez-Díaz \& J. Domínguez. 2006. Using FAME profiles for the characterization of animal wastes and vermicomposts. Soil Biology and Biochemistry. 38: 29932996.

Marinari, S., G. Masciandaro, B. Ceccanti \& S. Grego. 2000. Influence of organic and mineral fertilizers on soil biological and physical properties. Bioresource Technology. 72: 9-17

Mirza, M. S., W. Ahmad, F. Latif, J. Haurat, R. Bally, P. Normand \& K. A. Malik. 2001. Isolation, partial characterization, and the effect of plant growth-promoting bacteria (PGPB) on micro-propagated sugarcane in vitro. Plant and Soil. 237: 47-54.

Muscolo, A., F. Bovalo, F. Gionfriddo \& S. Nardi. 1999. Earthworm humic matter produces auxinlike effects on Daucus carota cell growth and nitrate metabolism. Soil Biology and Biochemistry. 31: 1303-1311.

Muscolo, A., M. Felici, G. Concheri \& S. Nardi. 1993. Effect of earthworm humic substances on esterase and peroxidase activity during growth of leaf explants of Nicotiana plumbaginifolia. Biology and Fertility of Soils. 15: 127-131.

Mylonas, V. A. \& C. B. Mccants. 1980. Effects of humic and fulvic acids on growth of tobacco. I. Root initiation and elongation. Plant and Soil. 54: 485-490.

Nardi, S., G. Arnoldi \& G. Dell'Agnola. 1988. Release of hormone-like activities from Alloborophora rosea and Alloborophora caliginosa feces. Journal of Soil Science. 68: 563-657.

Nardi, S., D. Pizzeghello, A. Muscolo \& A. Vianello. 2002. Physiological effects of humic substances on higher plants. Soil Biology and Biochemistry. 34: 1527-1536.

Nielson, R. L. 1965. Presence of plant growth substances in earthworms demonstrated by paper chromatography and the Went pea test. Nature. 208: 1113-1114.

Prabha, M. L., I. A. Jayraay, R. Jayraay \& D. S. Rao. 2007. Effect of vermicompost on growth parameters of selected vegetable and medicinal plants. Asian Journal of Microbiology, Biotechnology and Environmental Sciences. 9(2): 321-326.

Quaggiotti, S., B. Ruperti, D. Pizzeghello, O. Francioso, T. Vitaliano \& S. Nardi. 2004. Effect of low molecular size humic substances on nitrate uptake and expression of genes involved in nitrate transport in maize (Zea mays L.). Journal of Experimental Botany. 55: 803-813.

Reddy, M. V. \& K. Ohkura. 2004. Vermicomposting of rice-straw and its effects on sorghum growth. Tropical Ecology. 45(2): 327-331.

Rhee, J. A. van 1965. Earthworm activity and plant growth in artificial cultures. Plant and Soil. 22: 45-48.

Roberts, P., D. L. Jones \& G. Edwards-Jones. 2007. Yield and vitamin C content of tomatoes grown in vermicomposted wastes. Journal of the Science of Food and Agriculture. 87: 1957-1963.

Rodda, M. R. C., L. P. Canellas, A. R. Façanha, D. B. Zandonadi, J. G. M. Guerra, D. L. de Almeida \& G. A. de Santos. 2006. Improving lettuce seedling root growth and ATP hydrolysis with humates from Vermicompost. II- Effect of Vermicompost source. Revista Brasileira de Ciência do Solo. 30: 657-664.

Scheu, S. 1987. Microbial activity and nutrient dynamics in earthworm casts. Biology and Fertility of Soils. 5: 230-234. 
Schindler-Wessells, M. L., P. J. Bohlen, D. A. McCartney, S. Subler \& C. A. Edwards. 1996. Earthworm effects on soil respiration in corn agroecosystems receiving different nitrogen inputs. Soil Biology and Biochemistry. 29: 409-412.

Scott, M. A. 1988. The use of worm-digested animal waste as a supplement to peat in loamless composts for hardy nursery stock. Pp. 231-229. In: C. A. Edwards and E. F. Neuhauser (Eds.). Earthworms in waste and environmental management. SPB, The Hague.

Spain, A. V., P. Lavelle \& A. Mariotti. 1992. Stimulation of plant growth by tropical earthworms. Soil Biology and Biochemistry. 24: 1629-1633.

Springett, J. A. \& J. K. Syers. 1979. The effect of earthworm casts on ryegrass seedlings. Pp. 44-47. In: T. K. Crosby and R. P. Pottinger (Eds.). Proceedings of the 2nd Australasian Conference on Grassland Invertebrate Ecology. Government Printer, Wellington.

Stephens, P. M. \& C. W. Davoren. 1995. Effect of the lumbricid earthworm Aporrectodea trapezoids on wheat grain yield in the field, in the presence or absence of Rhizoctonia solani and Gaeumannomyces graminis var. tritici. Soil Biology and Biochemistry. 28: 561-567.

Sunil, K., C. R. Rawat, D. Shiva \& K. R. Suchit. 2005. Dry matter accumulation, nutrient uptake and changes in soil fertility status as influenced by different organic and inorganic sources of nutrients to forage sorghum (Sorghum bicolor). Indian Journal of Agricultural Science. 75(6): 340-342.

Syers, J. K. \& J. A. Springett. 1983. Earthworm ecology in grassland soils. Pp. 67-83. In: J. E. Satchell (Ed.). Earthworm ecology: from Darwin to vermiculture. Chapman \& Hall, London.

Tan, K. H. \& D. Tantiwiramanond. 1983. Effect of humic acids on nodulation and dry matter production of soybean, peanut, and clover. Soil Science Society of America Journal. 47: 1121-1124

Tiwari, S. C. \& R. R. Mishra. 1993. Fungal abundance and diversity in earthworm casts and in uningested soil. Biology and Fertility of Soils. 16: 131-134.

Trewavas, A. 2001. Urban myths of organic farming. Nature. 410: 409-410.

Tomati, U., A. Grappelli \& E. Galli. 1983. Fertility factors in earthworm humus. Pp. 49-56. In: U. Tomati and A. Grappelli (Eds.). Proceedings of the International Symposium on Agricultural and Environmental Prospects in Earthworm Farming. Tipolitografia Euromodena, Modena.

Tomati, U., A. Grappelli \& E. Galli. 1987. The presence of growth regulators in earthworm worked wastes. Pp. 423-436. In: A. M. Bonvicini Paglioi and P. Omodeo (Eds.). On Earthworms. Selected Symposia and Monographs 2. Mucchi Editore, Modena.

Tomati, U., A. Grappelli \& E. Galli. 1988. The hormone-like effect of earthworm casts on plant growth. Biology and Fertility of Soils. 5: 288-294.

Tomati, U., E. Galli, A. Grapppelli \& G. Dihena. 1990. Effect of earthworm casts on protein synthesis in radish (Raphanus sativum) and lettuce (Lactuca sativa) seedlings. Biology and Fertility of Soils. 9: 288-289.

Tomati, U. \& E. Galli. 1995. Earthworms, soil fertility and plant productivity. Acta Zoologica Fennica. 196: 11-14.

Valdrighi, M. M., A. Pera, M. Agnolucci, S. Frassinetti, D. Lunardi \& G. Vallini. 1996. Effects of compost-derived humic acids on vegetable biomass production and microbial growth within a plant (Cichorium intybus)-soil system: a comparative study. Agriculture, Ecosystems and Environment. 58: 133-144.

Vessey, J. K. 2003. Plant growth promoting rhizobacteria as biofertilizers. Plant and Soil. 255: 571-586.

Zaller, J. G. 2007. Vermicompost as a substitute for peat in potting media: Effects on germination, biomass allocation, yields and fruit quality of three tomato varieties. Scientia Horticulturae. 112: 191-199.

Zandonadi, D. B., L. P. Canellas \& A. Rocha Façanha. 2006. Indolacetic and humic acids induce lateral root development through a concerted plasmalemma and tonoplast $\mathrm{H}+$ pumps activation. Planta. 225(6): 1583-1595. 